\title{
Valorization of clinical trials from the Italian National Health Service perspective: definition and first application of a model to estimate avoided costs
}

\author{
Americo Cicchetti ${ }^{1}$, Domenico Addesso ${ }^{1}$, Filippo E. Leone ${ }^{1}$, Antonino Amato ${ }^{1}$, Luca Angerame ${ }^{1}$, Angelo D'Aversa ${ }^{2}$, \\ Mario Fraticelli ${ }^{3}$, Carlo Nicora ${ }^{4}$, Eleonora Sfreddo ${ }^{5}$, Mariangela Fumarola ${ }^{5}$, Roberta Porcino ${ }^{3}$, Gabriella Cocciolo ${ }^{6}$, \\ Simona $\mathbf{R e}^{6}$, Sergio Scaccabarozzi ${ }^{6}$ \\ ${ }^{1}$ Alta Scuola di Economia e Management dei Sistemi Sanitari, Università Cattolica del Sacro Cuore, Rome - Italy \\ ${ }^{2}$ Fondazione Policlinico Universitario "A. Gemelli" IRCCS, Rome - Italy \\ ${ }^{3}$ Azienda Socio-Sanitaria Territoriale (ASST) Papa Giovanni XXIII di Bergamo, Bergamo - Italy \\ ${ }^{4}$ Fondazione IRCCS Policlinico S. Matteo, Pavia - Italy \\ ${ }^{5}$ Fondazione per la Ricerca, Ospedale di Bergamo, Bergamo - Italy \\ ${ }^{6}$ Roche S.p.A., Monza (MB) - Italy
}

\begin{abstract}
Introduction: From the perspective of healthcare organizations and public health care systems, the value of a clinical trial can be assessed from a clinical and economical perspective. However, to date, there is no standardized model for systematically capturing the economic value of clinical trials at organizational and system levels. The aim of this study was to develop and test a methodology for estimating the avoided costs deriving from the management of patients as part of a clinical trial.

Methods: Our methodology is based on the assumption that the economic value of a clinical trial derives from 1) the funding received by the experimental site from a trial's sponsor, and from 2) the cost avoided by the experimental site with the treatment of patients within a study and not according to standard care by the experimental site.

Results: By applying the methodology to onco-hematological clinical trials conducted in two academic hospitals from 2011 to 2016, we demonstrate that savings between 2 million and 4 million euros were achieved over a five-year period. Thus, for every 1,000 euros invested by the pharmaceutical company into the clinical studies conducted at these hospitals, the hospitals saved on average 2,200 euros due to costs not incurred as a result of the trials.

Conclusions: The study has proposed and tested a methodology for estimating the economic value of clinical trials by taking into account avoided costs deriving from the treatment of patients enrolled in sponsored trials. The study has proposed a management tool for healthcare institutions to govern clinical trials.
\end{abstract}

Keywords: Avoided costs; Clinical trials; Healthcare management

\section{Introduction}

Clinical trials represent a milestone in the process of developing drugs and other health technologies. In addition to their experimental value, clinical trials generate value in

Received: January 3, 2020

Accepted: April 22, 2020

Published online: June 16, 2020

Corresponding author:

Americo Cicchetti

Director, Alta Scuola di Economia e Management dei Sistemi Sanitari (ALTEMS)

Università Cattolica del Sacro Cuore

Largo Francesco Vito, 1 - 00168 Rome - Italy

americo.cicchetti@unicatt.it themselves as biomedical technology translates from the initial concept to the clinical application. A clinical trial provides measurable "values" in different dimensions and from multiple perspectives. The patients, society, the national healthcare system, the healthcare professionals, and the drug industry, measure the "value" of a clinical trial differently according to the different aspects of safety and clinical efficacy they focus on. The economic and organizational impact at different levels of the experimental process are also relevant. A clinical trial can produce better health for patients, can improve the reputation of the investigators and their hospitals, can be strategic for a country's consideration as a host for clinical research, and can create economic value at the level of the healthcare system. Moreover, a clinical trial represents a value "per se", based on the quality of treatment provided to patients and on the physicians' compliance with the strict rules and standards of clinical trials. 
The $18^{\text {th }}$ National Report on Clinical Trials of Medicinal Products in Italy, published by the Italian Medicines Agency (AIFA) in 2019 (1), shows that the total number of clinical trials authorized in Italy increased from 564 in 2017 to 666 in 2018. Nevertheless, the total number of trials conducted in Italy between 2010 and 2018 remained steady. Despite the significant number of studies conducted, the attractiveness of conducting clinical trials in Italy compared to other countries appears to be low. The proportion of studies authorized in Italy compared to the European Union (EU) also appears to be stable, yet the total number of patients enrolled in clinical trials in Europe is declining.

The increasing cost of clinical research has significant implications for public health policies. For example, the increasing costs affect the propensity of pharmaceutical companies, as well as of government agencies, universities, and other non-profit organizations, to undertake clinical trials. In addition, rising clinical trial costs are a growing concern because of the economic risks associated with exploring potential applications of the new drugs (2). As a result, pharmaceutical companies are considering conducting clinical trials in countries where the trial costs are as much as $60 \%$ lower than the average costs incurred in European and North American countries (2). It is widely acknowledged that clinical studies provide an opportunity to demonstrate the safety and efficacy of both new and old drugs, including new or expanded indications of drugs already approved.

Pharmaceutical and medical device companies invest significant economic resources in clinical trials conducted at public and private hospitals worldwide. However, the economic implications of clinical trials for healthcare organizations are not limited to the payments received from the industry for the management of clinical trials. They include the costs of diagnostic tests and the assistance and time of the staff involved in a trial. Enrollment of patients in a clinical trial whether in the control or experimental arms - avoids burdening the institution with costs that would otherwise be incurred by providing standard of care treatment for patients (e.g., drug costs) in the absence of a trial. Therefore, measurement of clinical trial value holds interest for both researchers and healthcare providers worldwide. However, there is currently no standardized model for systematically capturing the economic value, at organizational and system levels, of trials assessing drugs and medical devices.

When Cicchetti et al. (3) performed a systematic literature review to identify articles on the value of clinical trials, a total of 854 studies were retrieved from PubMed and ISI Web of Science, of which seven were found to be specifically relevant. These papers recognized the usefulness and feasibility of calculating the economic value of clinical trials from the perspective of the payer (both private and public; regional and national) and of the hospitals where the trials were conducted. Some studies highlighted that the two components to be considered in order to fully understand the economic value of a clinical trial were 1 ) resources made available by sponsors through a fee-per-patient to cover the costs of diagnostic tests, health professional assistance, study staff, study materials etc., and 2) the value of the investigational drug. While the former component is easier to identify and calculate, the latter should be quantified considering the cost of the standard of care in the study's control arm, since generally the cost of an investigational drug is not known. The calculation of the latter component requires specific methodologies, and potential solutions for an appropriate calculation have been published. However, Tang et al. (4) demonstrated that there are specific methodological challenges due to the variability in drug doses and dosing regimens. Moreover, Drug Cost Avoidance (DCA) associated with the cost of an investigational drug and Pathology Cost Avoidance (PCA) associated with diagnostic costs need to be considered. A limitation of Tang's study, as well as of others, is that calculations of the global economic impact of a trial are based on the number of patients potentially participating in a trial (planned patients), and not on the number of patients who have been enrolled and have completed the study.

The aim of the current study is to develop and test a methodology for estimating the economic value of conducting clinical trials by taking into account the avoided costs for the treatment of the enrolled patients. With this goal, we calculated the avoided costs for the enrolled and the planned patients of a set of trials conducted in the settings of a public and a private hospital in Italy. We demonstrated that this methodology provides a better estimate of the "leverage effect" of a clinical trial, defined as the ratio between the avoided costs and the fees paid by sponsors to a hospital for treating patients participating in a trial. We suggest that this methodology should be useful for estimating the economic value of a clinical trial from both the hospital perspective and the perspective of a regional health authority.

\section{Methods \\ Participants}

This study involved a multidisciplinary working group comprising pharmacists, health economists, healthcare managers, and physicians from two academic medical centers in Italy and from the pharmaceutical company Roche. ALTEMS, a Graduate School of Health Economics and Management (Università Cattolica del Sacro Cuore, Rome), provided methodological support for the identification of a common approach to be applied to two academic medical centers (AMCs) in Italy: Fondazione Policlinico Universitario "A. Gemelli" of Rome (FPG) and Azienda Socio Sanitaria Territoriale Papa Giovanni XXIII of Bergamo (ASST BG).

Both AMCs had experience in conducting clinical research. The clinicians/researchers at both AMCs had previously participated in designing and conducting phase 2 and phase 3 clinical trials. Moreover, both centers had a dedicated staff for the management of clinical trials. FBG has specifically established a Clinical Trial Center (CTC). This CTC was previously a branch of the hospital, whilst now is an independent business company owned by FPG. ASST BG has established a Research Foundation (named "FROM", Fondazione per la Ricerca, Ospedale Papa Giovanni XXIII), which is dedicated to the management of clinical trials and other clinical and translational research.

A database was created which contains the data from all clinical trials of new onco-hematologic treatments sponsored 
by Roche at FPG and ASST BG between 2011 and 2016. The clinical trials in the database were started and closed (e.g., all the enrolled patients had completed the follow-up phase) between 2011 and 2016. Funding data were derived from the economic agreements signed by the two AMCs and the sponsor, Roche S.p.A., and detailed the turnover of FPG and ASST BG for the trials carried out during the period of interest.

\section{Indicators examined}

Two indicators were evaluated: cumulative funding per study and total in a given period of time. This indicator was calculated adding the invoices issued and received for the patients enrolled and treated in clinical trials started within year $t$ and completed within year $t+n$ (e.g., 2011-2016). Data were drawn from the relevant financial statements. To establish a value for the net financial contribution given to a hospital by a sponsor, both costs and revenues of each clinical trial were taken into account (5). However, the accounting information made available from the hospitals was incomplete. Therefore, we adopted the approach outlined above to obtain a reasonable proxy of the financial contribution provided by the sponsor to the hospitals.

The second indicator we calculated, the avoided cost, was the sum of drug costs incurred for treating patients in the experimental and control arms if these patients were not included in the clinical study and were treated according to the standard clinical practice. This indicator highlights the savings, in terms of cost reduction, resulting from the administration of experimental drugs to treat the patients enrolled in a clinical trial, whether they are in the experimental arm or in the control arm. Whenever possible, it was assumed that the control arm was receiving the standard of care. Moreover, if more than one type of treatment was available, the less expensive treatment was considered.

\section{Leverage effect}

Both indicators were used to calculate a "leverage" effect. This effect considers the funding provided by an industry for the experimental investigation and the total avoided cost as follows: cumulative funding per study + total avoided cost/ cumulative funding per study. This leverage indicator can have any value greater than 1 and represents a multiplier factor, or the power of the "leverage" effect generated by a sponsor's investment. For example, if the indicator has a value of 2 and the sponsor invested 1,000 euros as "compensation" payment to an AMC, the AMC saves 2,000 euros for costs not incurred to purchase health technologies, staff assistance, and treatments (e.g., drugs) as required if those patients were not included in the study.

\section{Valorization model}

A model for valorization of total avoided costs was generated by comparing (and subsequently validating) the model proposed in the "Valorization Of clinical Research" (ValOR) project by Roche (Fig. 1) with the models used by FPG and ASST BG. The ValOR model defines the value produced by clinical research activities performed in the hospital as the sum of two components: 1) the funding received by the experimental site (e.g., cumulative revenues from financing of the investigation, which include all costs for patients' management), and 2) the total cost avoided by the experimental site (e.g., the estimate of costs avoided by the NHS since the sponsor/drug company would bear the cost of drug therapies for the patients enrolled in the clinical study).

To calculate the two components of the ValOR model, the following factors were taken into account:

- the total financing foreseen (fees \& grants) as indicated in the contract of each clinical trial;

- the average value of the experimental treatment arms, if two or more were involved;

- the economic value, based on the number of the enrolled patients;

- the total per patient (no pro rata) provided at the time of enrollment according to the contract;

- it was assumed that patients completed the treatment according to the study protocol (no dropouts/early withdrawals);
Valorization of clinical studies from the hospital perspective

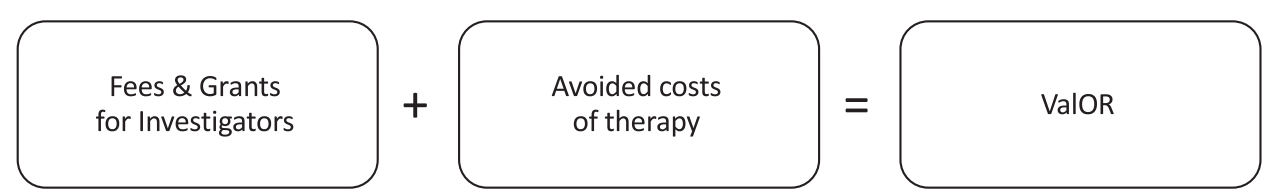

Study fees paid to the institution to cover assistance, diagnostic tests, study staff, material,

Estimate of treatment costs not incurred by the NHS (NHS Savings)

etc.

Fig. 1 - Representation of the Roche model ValOR (Valorization Of Clinical Research) (Authors' elaboration). 
- treatment duration, that was calculated based on the period defined by the protocol (e.g., 1 year of therapy). If the duration was not clearly defined (e.g., treatment until disease progression), the duration of treatment was estimated according to literature on the standard therapy received by the control arm.

To quantify the avoided cost, the following assumptions were made:

- treatment duration was calculated according to literature on the control arm therapy;

- treatment provided or reimbursed was valued, including what the patient would have received if not participating in the protocol (e.g., control drug for randomized trials or backbone therapy for single arm trials). The cost of investigational drugs was not valued;

- treatment duration was expressed in cycles or in time units (months or days) depending on frequency;

- when more than one treatment option was available, the least expensive option was valued.

To quantify the avoided cost of the drugs that patients would have received regardless of participation in the study, and which were provided or reimbursed by the sponsor as comparators or backbone therapy, the unit drug cost (exfactory price) was obtained from multiple databases (drug industry databases or public sources). Discounts or agreements defined during the negotiation phase with health authorities (e.g., payment by results, cost sharing) were not taken into consideration. If the dose of a given drug was based on body weight in kilograms $(\mathrm{kg})$, or on body surface in square meters $\left(\mathrm{m}^{2}\right)$, the value obtained was multiplied by the following standard coefficients: 70 , according to the average weight of an individual (either male or female); or 1.7, according to the average body area of an individual (either male or female), respectively. Once the unit cost of the drug at the dosage administered in the clinical trial was calculated, it was multiplied by the estimated number of days it was administered and by the number of patients receiving the drug. In studies with a single experimental treatment arm (and in the absence of a backbone therapy), or in studies of an experimental drug versus placebo, it was assumed that the value of the avoided cost was zero. This was a conservative assumption and was intended to prevent introducing the complexity related to the standard therapy that the patient would have received if treated according to the standard clinical practice.

\section{Results}

We identified 29 studies sponsored by Roche conducted at the two Italian AMCs (FPG and ASST BG). Active recruitment for these studies started in 2011, and recruitment was discontinued by December 31, 2016. A total of 189 patients were enrolled in these studies involving onco-hematologic patients: there were more oncological than hematological studies (Tables I and II). Overall, oncological + hematological

TABLE I - Studies sponsored by Roche at FPG started and closed between 2011 and 2016*.

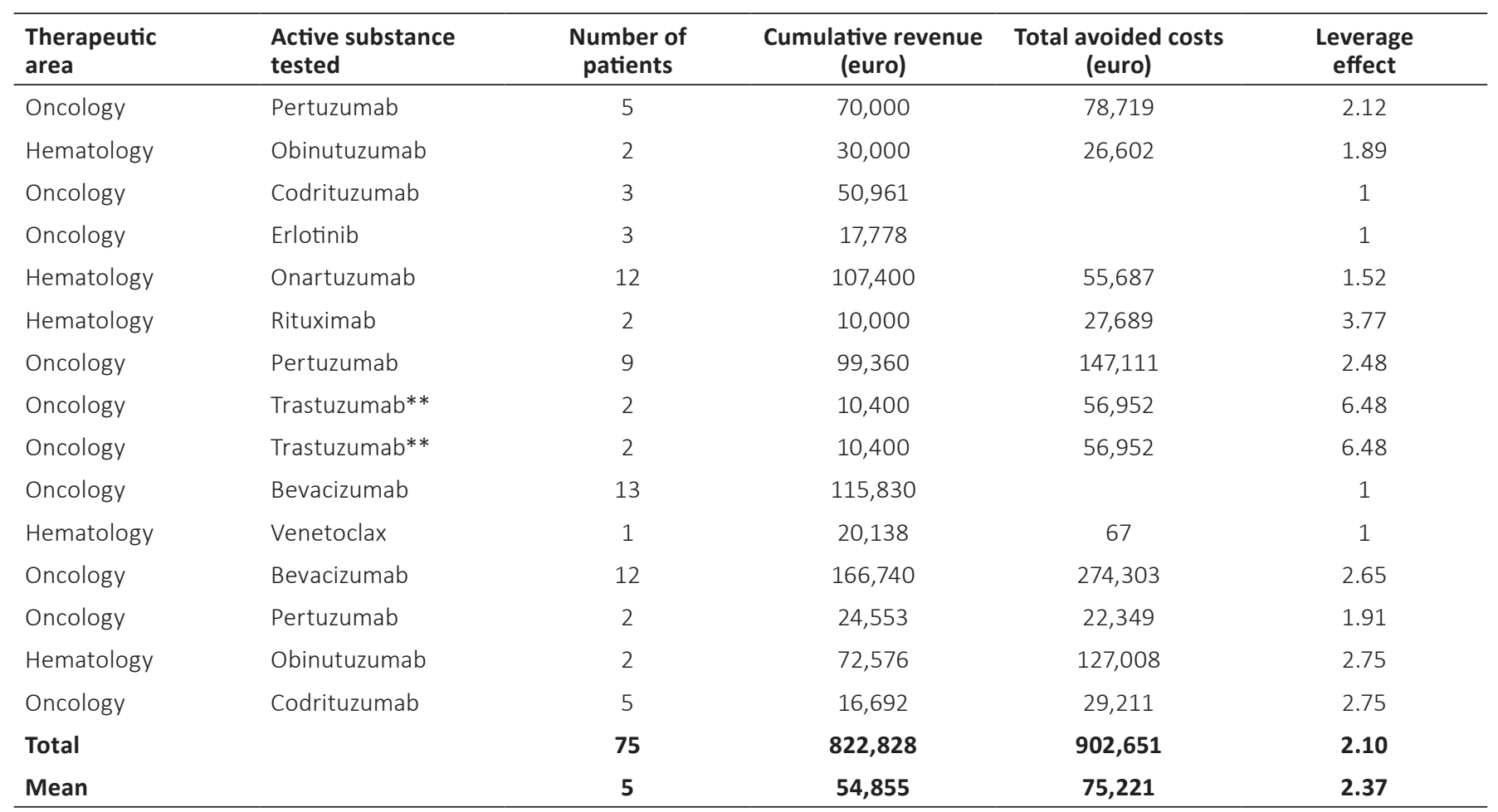

*All the enrolled patients completed the follow-up phase in this period.

**This trial was conducted by two research teams. 
TABLE II - Studies sponsored by Roche at ASST BG started and closed between 2011 and 2016*.

\begin{tabular}{|c|c|c|c|c|c|}
\hline $\begin{array}{l}\text { Therapeutic } \\
\text { area }\end{array}$ & $\begin{array}{l}\text { Active substance } \\
\text { tested }\end{array}$ & $\begin{array}{c}\text { Number of } \\
\text { patients }\end{array}$ & $\begin{array}{c}\text { Cumulative revenue } \\
\text { (euro) }\end{array}$ & $\begin{array}{c}\text { Total avoided } \\
\text { costs (euro) }\end{array}$ & $\begin{array}{c}\text { Leverage } \\
\text { effect }\end{array}$ \\
\hline Oncology & Cabazitaxel & 7 & 19,250 & 121,569 & 7.32 \\
\hline Oncology & Vemurafenib & 37 & 148,000 & 153,605 & 2.04 \\
\hline Oncology & Trastuzumab emtansine & 7 & 78,740 & 27,775 & 1.35 \\
\hline Oncology & Pertuzumab & 10 & 105,000 & 432,370 & 5.12 \\
\hline Oncology & Custirsen & 10 & 87,840 & 35,227 & 1.40 \\
\hline Oncology & LY2157299 & 1 & 9,891 & 2,146 & 1.22 \\
\hline Oncology & Nivolumab & 5 & 99,442 & 17,325 & 1,17 \\
\hline Hematology & Blinatumomab & 9 & 80,874 & 756 & 1.01 \\
\hline Hematology & Sapacitabine & 1 & 5,018 & 17,094 & 4.41 \\
\hline Total & & 114 & 861,918 & $1,094,603$ & 2.27 \\
\hline Mean & & 8.14 & 61,566 & 78,186 & 2.87 \\
\hline
\end{tabular}

sc = subcutaneous.

*All the enrolled patients completed the follow-up phase in this period.

studies sponsored by Roche accounted for $46 \%$ and $55 \%$ of the total studies conducted by FPG and ASST BG, respectively. The total cumulative revenue (Fees \& Grants for Investigators] for the two AMCs was 2.5 million euros, while the total avoided cost for therapy was 3.5 million euros. Thus, the recorded avoided costs totaled approximately 330 thousand euros over the five-year period analyzed. It should be noted that in our analysis the avoided cost did not include extra-budgetary costs, costs of medical research staff and nurses, equipment, etc. The only cost included in the avoided cost was the cost of drug therapies administered during the investigation.

When the leverage effect (or multiplier effect) was calculated, a correspondence between the values recorded by FPG and ASST BG was observed. In both cases, a leverage effect is observed and appears to be relevant. For example, for each euro FPG received from the financing industry, the avoided costs were $2.37 €$; as for ASST BG, $2.87 €$ were saved for each euro received.

\section{Discussion}

In this study, we proposed and tested a methodology for estimating the economic value of clinical trials by taking into account the avoided costs deriving from treatment of patients enrolled in sponsored trials. In the last few years, awareness of the value of clinical trials was growing at both drug industry and health institution levels. This interest is also related to the European Regulation no. 536/2014 (6). This regulation addresses the competition between European and other countries to attract investments for investigational activities, offering new opportunities to the Italian NHS. Furthermore, it has prompted the Italian NHS organizations to equip themselves with new tools and solutions to increase the attractiveness of the Italian healthcare system for conducting clinical trials. A keystone is certainly the promotion of a more systematic involvement of health organizations as "managing bodies" of clinical trials.

The primary aim of this study was to propose a management tool for healthcare organizations to effectively govern clinical trials. Our secondary aim was to generate greater awareness of clinical trial economic value in order to incentivize their prioritization among healthcare activities. The results of our study have scientific, managerial, and institutional implications.

From a scientific point of view, this study contributes to the growing body of literature on the economic value of clinical trials (3). In our study, the analysis of the data set of multidisciplinary and multi-institutional clinical trials offered the opportunity of developing and testing a methodology for quantifying the full economic value of clinical trials. From a managerial point of view, our study provides a management tool for those responsible for clinical trial management in the NHS setting. Clinical trial optimal management requires a combination of available resources, competencies, and operative mechanisms. These aspects, as well as the characteristics of organizational solutions implemented in Italy for the management of clinical trials - such as Clinical Trial Office/Centers - have previously been described 
and analyzed $(3,5,7)$. In the current study, the focus is on the economic impact of clinical trials. Specifically, a methodology was described for the calculation of two key indicators (cumulative financing and total avoided costs) which can be easily introduced in hospital dashboards to better measure the contribution of clinical research to the financial sustainability of research hospitals. Finally, from an institutional point of view, our analysis confirms and supports the observation that the "fees", granted to the hospitals by pharmaceutical companies for conducting clinical studies, represent only part of the economic value potentially generated by clinical research performed in the hospital setting. Our analysis can also help regional health authorities to clearly identify which hospital in a regional network is generating value for the patients without bearing the related costs. From this perspective, information regarding cumulative financing and avoided costs should be included in hospital financial statements, and taken into consideration when defining budgetary allocations at the regional level.

When we focused on the data related to oncological treatments provided by the two AMCs, we observed that avoided costs account for the largest proportion of the economic advantages of clinical trials. The avoided costs were approximately three times the revenue generated by the sponsorship agreements for the investigational studies. Our results highlight that research activities performed between 2011 and 2016 in the onco-hematological setting enabled cost-savings of approximately 2 million euros overall. Despite these findings cannot be fully generalized, if our model is applied to all onco-hematological studies conducted by Roche Italy between 2011 and 2016, the total per-patient fees amounted to 66.6 million euros, while the total savings related to drug therapies were 84.6 million euros. Therefore, the total economic impact on the Italian health system would be 151.3 million euros, with a leverage effect of 2.2 for this set of studies. Applying this leverage effect to the total number of clinical trials recorded in the Osmed 2015 Report (8) which includes 86 hospital trusts/academic medical centers and 48 public and private IRCCS - the potential total avoided costs at national level would range from 320 to 360 million euros/year. This amount only derives from onco-hematological studies, and calculations were performed with a conservative approach. Overall, these results strongly support the recommendation that hospital administrators should prioritize clinical trials, rather than other opportunities, as source of funding and cost-savings; investments in terms of organization and additional human resources dedicated to clinical trials should be strongly considered.

Our results are consistent with those of other studies carried out at national level in Italy $(5,7,9,10)$ : clinical trials funded by sponsors that often supply the drugs for clinical research free of charge, translate into significant economic savings for the research hospitals. Funding received for each enrolled patient provides surplus funds for the involved healthcare provider. The economic implications at system level appear to be significant, as both the experimental drugs and the active comparators in the control arms are provided by the sponsor of the clinical trial. This observation leads to a further "accounting" consideration. Currently, avoided cost are not included in healthcare organization financial statements. Moreover, savings due to avoided costs are not reported among the income positive components of hospital financial statements, although these savings contribute to the net economic result. This aspect needs further investigation. We would also suggest that avoided costs should be taken into account when health organizations are defining budgetary resource allocations to the pharmaceutical expenditure. If these avoided costs are consolidated and historicized, the support and commitment to participation in clinical research projects would improve.

Our study has some limitations. First, the analysis was conducted on data provided by only two AMCs in Italy, however large in size. In addition, the accounting systems in place at FPG and ASST BG, did not allow us to analyze all the available indicators. Moreover, our model for quantifying avoided cost was run using data from trials sponsored by one pharmaceutical company (Roche) and carried out in a specific clinical area, i.e. the onco-hematological setting. Consequently, the leverage effect, as well as the avoided costs per patient, are inevitably affected by the conventional treatment provided to patients of that specific clinical area. Therefore, our results cannot be fully generalized to all teaching hospitals in Italy. To overcome these limitations, model input data should be collected from multiple hospitals and be related to several clinical areas (e.g., cardiology, neurology, diabetes, etc.). The analysis of data for different hospitals and diseases would improve our understanding of how clinical trials could be supported by multiple sponsors, and how contextual factors, such as the size and characteristics of a hospital, the clinical area of investigation, and the characteristics of the sponsor, can impact on both the leverage effect and the avoided cost per patient.

\section{Conclusion}

This study was made possible through the collaboration among two healthcare institutions (FPG and ASST BG), an academic research center (ALTEMS), and a drug company (Roche). These stakeholders have different interests and perspectives, and their collaboration allowed us to demonstrate the value of clinical trials beyond the widely acknowledged importance of collaborations between public and private sectors and between clinical practice and academic setting. We hope that our work will bring to health authorities attention the importance of management control and accounting systems to increase the opportunities for the Italian NHS to conduct clinical trials, which not only hold the potential for improved health care, but also create economic value at both organizational and system levels.

\section{Disclosures}

Financial support: This research has been supported by an unconditional grant from Roche S.p.A. paid to ALTEMS, Graduate School of Health Economics and Management (Università Cattolica del Sacro Cuore). Conflict of interest: AM and DA report grants from ROCHE during the conduct of the study; $C N$ reports a non-financial support from Roche to perform this specific study. FL, $A A$, LA, AD, MF, ES, MF, RP, GC, SR, and SS have nothing to disclose. 


\section{References}

1. Agenzia Italiana del Farmaco (2019). La Sperimentazione Clinica dei Medicinali in Italia. $18^{\circ}$ Rapporto Nazionale, Roma.

2. Collier R. Rapidly rising clinical trial costs worry researchers. CMAJ 2009;180(3):277-8.

3. Cicchetti A, Addesso D, Amato $A$ et al. Valorizzazione delle sperimentazioni cliniche nella prospettiva del SSN: Definizione di un modello per una stima dei costi evitati. Milano: Edra, 2018.

4. Tang PA, Hay AE, O'Callaghan $\mathrm{CJ}$ et al. Estimation of drug cost avoidance and pathology cost avoidance through participation in NCIC Clinical Trials Group phase III clinical trials in Canada. Curr Oncol 2016;23(S1):S7-13.

5. Cavazza, M, Costa F, Jommi C. Organizzazione e gestione delle sperimentazioni cliniche. Milano: EGEA, 2016.

6. Regulation (EU) no. 536/2014 of the European Parliament and of the Council of 16 April 2014 on clinical trials on medicinal

products for human use, and repealing Directive 2001/ 20/EC.

7. Cavazza M, Bertolani A, Jommi C. La ricerca clinica in Italia: quanto, come viene finanziata e i suoi effetti sul sistema. In: Gussoni G. (ed.). II valore della ricerca clinica indipendente in Italia. Milano: EDRA, 2019.

8. Agenzia Italiana del Farmaco. L'uso dei farmaci in Italia. Rapporto OSMED, 2016.

9. Grossi F, Genova C, Gaitan ND et al. Free drugs in clinical trials and their potential cost saving impact on the National Health Service: a retrospective cost analysis in Italy. Lung Cancer 2013;81:236-40.

10. Spandonaro F, D’Angela D. Un caso studio sulla valutazione degli impatti generati dalle aziende farmaceutiche in una prospettiva pubblica. Roma: Centro Studi Crea Sanità, Università di Tor Vergata, 2015. 